\title{
Existence of nonconstant periodic
} solutions for a class of second-order systems with $p(t)$-Laplacian

\author{
Yukun $\mathrm{An}^{1^{*},}$, Yuanfang $\mathrm{Ru}^{2,3}$ and Fanglei Wang ${ }^{3}$
}

\section{${ }^{*}$ Correspondence:}

anykna@nuaa.edu.cn

${ }^{1}$ College of Science, Nanjing

University of Aeronautics and

Astronautics, Nanjing, 210016, P.R.

China

Full list of author information is

available at the end of the article

\begin{abstract}
In this paper, we investigate a class of second-order $p(t)$-Laplacian systems with local 'superquadratic' potential. By using the generalized mountain pass theorem, we obtain an existence result for nonconstant periodic solutions.
\end{abstract}

Keywords: $p(t)$-Laplacian; generalized Sobolev space; generalized mountain pass theorem; periodic solution

\section{Introduction}

This paper is concerned with the existence of periodic solutions for the following $p(t)$ Laplacian system:

$$
\left\{\begin{array}{l}
\left(\left|u^{\prime}(t)\right|^{p(t)-2} u^{\prime}(t)\right)^{\prime}+\nabla F(t, u(t))=0, \quad t \in[0, T] \\
u(0)-u(T)=u^{\prime}(0)-u^{\prime}(T)=0
\end{array}\right.
$$

where $T>0, u \in R^{N} . F(t, u)$ and $p(t)$ satisfy the following conditions:

$\left(F_{0}\right) F:[0, T] \times R^{N} \rightarrow R$ is measurable and $T$-periodic in $t$ for each $u \in R^{N}$ and continuously differentiable in $u$ for a.e. $t \in[0, T]$, and there exist $a \in C\left(R^{+}, R^{+}\right)$and $b \in L^{1}\left([0, T], R^{+}\right)$such that

$$
|F(t, u)| \leq a(|u|) b(t), \quad \nabla|F(t, u)| \leq a(|u|) b(t)
$$

for all $u \in R^{N}$ and a.e. $t \in[0, T]$.

(P) $p(t) \in C\left([0, T], R^{+}\right), p(t)=p(t+T)$ and

$$
1<p^{-}:=\min p(t) \leq p^{+}:=\max p(t)<+\infty .
$$

The $p(t)$-Laplacian system can be applied to describe the physical phenomena with 'pointwise different properties' which first arose from the nonlinear elasticity theory (see [1]).

\section{Springer}


If $p(t)=p$ is a constant, system (1) reduces to the $p$-Laplacian system

$$
\left\{\begin{array}{l}
\left(\left|u^{\prime}(t)\right|^{p-2} u^{\prime}(t)\right)^{\prime}+\nabla F(t, u(t))=0, \quad t \in[0, T] \\
u(0)-u(T)=u^{\prime}(0)-u^{\prime}(T)=0
\end{array}\right.
$$

Especially, when $p=2$, system (1) or (2) becomes the well-known second-order Hamiltonian system

$$
\left\{\begin{array}{l}
u^{\prime \prime}(t)+\nabla F(t, u(t))=0, \quad t \in[0, T] \\
u(0)-u(T)=u^{\prime}(0)-u^{\prime}(T)=0
\end{array}\right.
$$

In 1978, Rabinowitz [2] published his pioneer paper on the existence of periodic solutions for problem (3) under the following Ambrosetti-Rabinowitz superquadratic condition:

(AR) There exist $\mu>2$ and $L^{*}>0$ such that

$$
0<\mu F(t, u) \leq(\nabla F(t, u), u)
$$

for all $|u| \geq L^{*}$ and a.e. $t \in[0, T]$.

From then on, many researchers have tried to replace the Ambrosetti-Rabinowitz (shortened $A R$ ) condition by other superquadratic conditions. Some new superquadratic conditions under which there exist periodic solutions for problem (3) have been discovered in literature, see, for example, the references [3-5]. In [5], the authors obtained the following existence theorem for (3) under the 'local superquadratic conditions'.

Theorem A ([5], Theorem 1.1) Suppose that $F(t, u)$ satisfies $\left(F_{0}\right)$ and the following conditions:

$\left(V_{1}\right) F(t, u) \geq 0$ for all $t \in[0, T]$ and $u \in R^{N}$;

$\left(V_{2}\right)$ There are constants $m>0$ and $\alpha \leq \frac{6 m^{2}}{T^{2}}$ such that $F(t, u) \leq \alpha$ for all $u \in R^{N},|u|<m$ and a.e. $t \in[0, T]$.

$\left(V_{3}\right)$ There are constants $\mu>2,1 \leq \gamma<2, G>0$ and the function $d(t) \in L^{1}\left([0, T], R^{+}\right)$such that

$$
\begin{gathered}
\qquad \mu F(t, u) \leq(\nabla F(t, u), u)+d(t)|u|^{\gamma} \\
\text { for all } u \in R^{N},|u| \geq G \text { and a.e. } t \in[0, T] .
\end{gathered}
$$

$\left(V_{4}\right)$ There exist a constant $M>0$ and a subset $E$ of $[0, T]$ with meas $(E)>0$ such that

(a) $\liminf _{|u| \rightarrow \infty} \frac{F(t, u)}{|u|^{2}}>0$ uniformly for a.e. $t \in E$;

(b) $d(t) \leq M$ for a.e. $t \in E$.

Then problem (3) has at least one nonconstant T-periodic solution.

Recently, in [6], the authors extended the above result of [5] to system (2) and got the following theorem for (2). 
Theorem B ([6], Theorem 1.4) Suppose that $F(t, u)$ satisfies $\left(F_{0}\right),\left(V_{1}\right)$ and the following conditions:

$\left(H_{1}\right) \liminf _{|u| \rightarrow 0} \frac{F(t, u)}{|u|^{p}}=0$ uniformly for a.e. $t \in[0, T]$;

$\left(H_{2}\right)$ There are constants $\mu>p, G>0$ and the function $d(t) \in L^{1}([0, T], R)$ such that

$$
\mu F(t, u)-(\nabla F(t, u), u) \leq d(t)|u|^{p}
$$

for all $u \in R^{N},|u| \geq G$ and a.e. $t \in[0, T]$, and

$$
\limsup _{|u| \rightarrow \infty} \frac{\mu F(t, u)-(\nabla F(t, u), u)}{|u|^{p}} \leq 0
$$

uniformly for a.e. $t \in[0, T]$;

$\left(H_{3}\right)$ There exists a subset $E$ of $[0, T]$ with meas $(E)>0$ such that

$$
\liminf _{|u| \rightarrow \infty} \frac{F(t, u)}{|u|^{p}}>0
$$

uniformly for a.e. $t \in E$.

Then problem (2) has at least one nonconstant T-periodic solution.

On the other hand, the $p(t)$-Laplacian system has been studied by many authors in the last two decades, see, for example, [7-11] and the references cited therein. In [8], by using linking methods, the authors obtained an existence result under the $A R$ condition as follows.

Theorem C ([8], Theorem 4.1) Suppose that conditions $(P)$ and $\left(F_{0}\right)$ hold and $F(t, u)$ satisfies the following conditions:

$\left(A_{1}\right) F(0,0)=0$ and $F(t, u) \geq 0$ for all $t \in[0, T]$ and $u \in R^{N}$;

$\left(A_{2}\right)$ There are constants $\mu>P^{+}$and $G>0$ such that

$$
\begin{gathered}
\qquad \mu(t, u) \leq(\nabla F(t, u), u) \\
\text { for all } u \in R^{N},|u| \geq G \text { and a.e. } t \in[0, T] ;
\end{gathered}
$$

$\left(A_{3}\right)$ There exist $v>P^{+}$and $g \in C([0, T], R)$ such that

$$
\limsup _{|u| \rightarrow \infty} \frac{F(t, u)}{|u|^{v}} \leq|g(t)| \text {. }
$$

Then problem (1) has at least one periodic solution.

Moreover, in [12-14], the authors studied a superlinear elliptic equation with $p(x)$ Laplacian without the $A R$ condition and obtained some existence results.

Motivated by the papers $[3,5,6,8,12]$, we aim in this paper to study the existence of nonconstant periodic solutions of system (1) with local 'superquadratic' potential and without the $A R$ condition. We get an existence result which generalizes the above Theorem A and Theorem B and extends Theorem $C$. That is the following theorem. 
Theorem 1 Suppose that conditions $(P)$ and $\left(F_{0}\right)$ hold and, in addition, $F(t, u)$ satisfies the following conditions:

(F) $F(t, u) \geq 0$ for all $t \in[0, T]$ and $u \in R^{N}$;

$\left(F_{2}\right) \liminf _{|u| \rightarrow 0} \frac{F(t, u)}{|u|^{p^{+}}}=0$ uniformly for a.e. $t \in[0, T]$;

$\left(F_{3}\right)$ There are constants $\mu>p^{+}, G>0$ and the function $d(t) \in L^{1}([0, T], R)$ such that

$$
\mu F(t, u)-(\nabla F(t, u), u) \leq d(t)|u|^{p^{-}}
$$

for all $u \in R^{N},|u| \geq G$ and a.e. $t \in[0, T]$, and

$$
\limsup _{|u| \rightarrow \infty} \frac{\mu F(t, u)-(\nabla F(t, u), u)}{|u|^{p^{-}}} \leq 0
$$

uniformly for a.e. $t \in[0, T]$;

$\left(F_{4}\right)$ There exists a subset $\Omega$ of $[0, T]$ with meas $(\Omega)>0$ such that

$$
\liminf _{|u| \rightarrow \infty} \frac{F(t, u)}{|u|^{p^{+}}}>0
$$

uniformly for a.e. $t \in \Omega$.

Then problem (1) has at least one nonconstant $T$-periodic solution.

Example Set $p(t)=\frac{5}{2}+\sin \left(\frac{2 \pi}{T} t-\frac{\pi}{2}\right)$, then $p(t)$ satisfies condition $(P)$ and $p^{-}=\frac{3}{2}, p^{+}=\frac{7}{2}$. Let

$$
\begin{aligned}
& \psi(t)= \begin{cases}\sin \left(\frac{2 \pi}{T} t\right), & t \in\left[0, \frac{T}{2}\right], \\
0, & t \in\left[\frac{T}{2}, T\right],\end{cases} \\
& \phi(u)= \begin{cases}|u|^{4}, & |u| \leq 1, u \in R^{N}, \\
\frac{16}{5}|u|^{\frac{5}{4}}, & |u|>1, u \in R^{N},\end{cases} \\
& F(t, u)=\psi(t)|u|^{4}+\phi(u), \quad t \in[0, T], u \in R^{N} .
\end{aligned}
$$

It is clear that $\left(F_{1}\right)$ and $\left(F_{2}\right)$ hold. Let $\mu=4, G=1$, then $\left(F_{3}\right)$ holds. And take $\Omega=\left[\frac{T}{8}, \frac{3 T}{8}\right]$, then $\left(F_{4}\right)$ holds for $t \in \Omega$. Therefore, $F$ satisfies all the conditions of our Theorem 1 . Moreover, it is easy to verify that the function $F(t, u)$ does not satisfy the $A R$ condition $A_{2}$ in Theorem $\mathrm{C}$ for $t \in\left[\frac{T}{2}, T\right]$.

\section{Preliminaries}

For the reader's convenience, we first give some necessary background knowledge and propositions concerning the generalized Lebesgue-Sobolev spaces. We can refer the reader to $[8,15-19]$. In the following, we use $|\cdot|$ to denote the Euclidean norm in $R^{N}$.

Let $p(t)$ satisfy condition $(P)$ and define

$$
L^{p(t)}\left([0, T] ; R^{N}\right)=\left\{u \in L^{1}\left([0, T] ; R^{N}\right): \int_{0}^{T}|u|^{p(t)} d t<\infty\right\}
$$


An et al. Boundary Value Problems (2017) 2017:170

Page 5 of 15

with the norm

$$
|u|_{L^{p(t)}}=|u|_{p(t)}=\inf \left\{\lambda>0: \int_{0}^{T}\left|\frac{u}{\lambda}\right|^{p(t)} d t \leq 1\right\}
$$

Define

$$
C_{T}^{\infty}=C_{T}^{\infty}\left(R ; R^{N}\right)=\left\{u \in C^{\infty}\left(R ; R^{N}\right): u \text { is } T \text {-periodic }\right\}
$$

For $u \in L^{1}\left([0, T] ; R^{N}\right)$, if there exists $v \in L^{1}\left([0, T] ; R^{N}\right)$ satisfying

$$
\int_{0}^{T} v \varphi d t=-\int_{0}^{T} u \varphi^{\prime} d t, \quad \forall \varphi \in C_{T}^{\infty}
$$

then $v$ is called the $T$-weak derivative of $u$ and is denoted by $u^{\prime}$. Define

$$
W_{T}^{1, p(t)}\left([0, T] ; R^{N}\right)=\left\{u \in L^{p(t)}\left([0, T] ; R^{N}\right): u^{\prime} \in L^{p(t)}\left([0, T] ; R^{N}\right)\right\}
$$

with the norm

$$
\|u\|_{W_{T}^{1, p(t)}}=\|u\|=|u|_{p(t)}+\left|u^{\prime}\right|_{p(t)} .
$$

For $u \in W_{T}^{1, p(t)}\left([0, T] ; R^{N}\right)$, let

$$
\bar{u}=\frac{1}{T} \int_{0}^{T} x(s) d s, \quad \tilde{u}(t)=u(t)-\bar{u}
$$

and

$$
\widetilde{W}_{T}^{1, p(t)}\left([0, T] ; R^{N}\right)=\left\{x \in W_{T}^{1, p(t)}\left([0, T] ; R^{N}\right): \int_{0}^{T} x(s) d s=0\right\},
$$

then

$$
W_{T}^{1, p(t)}\left([0, T] ; R^{N}\right)=\widetilde{W}_{T}^{1, p(t)}\left([0, T] ; R^{N}\right) \oplus R^{N}
$$

In the following we use $L^{p(t)}, W_{T}^{1, p(t)}, \widetilde{W}_{T}^{1, p(t)}$ to denote $L^{p(t)}\left([0, T] ; R^{N}\right), W_{T}^{1, p(t)}\left([0, T] ; R^{N}\right)$, $\widetilde{W}_{T}^{1, p(t)}\left([0, T] ; R^{N}\right)$, respectively.

Proposition 1 ([7]) For $u \in L^{p(t)}$, one has

(1) $|u|_{p(t)}<1(=1 ;>1) \Leftrightarrow \int_{0}^{T}|u(t)|^{p(t)} d t<1(=1 ;>1)$;

(2) $|u|_{p(t)}>1 \Rightarrow|u|_{p(t)}^{p^{-}} \leq \int_{0}^{T}|u(t)|^{p(t)} d t \leq|u|_{p(t)}^{p^{+}}$;

$|u|_{p(t)}<1 \Rightarrow|u|_{p(t)}^{p^{+}} \leq \int_{0}^{T}|u(t)|^{p(t)} d t \leq|u|_{p(t)}^{p^{-}}$ 


$$
\text { (3) } \begin{aligned}
|u|_{p(t)} \rightarrow 0 & \Leftrightarrow \int_{0}^{T}|u(t)|^{p(t)} d t \rightarrow 0 ; \\
|u|_{p(t)} \rightarrow \infty & \Leftrightarrow \int_{0}^{T}|u(t)|^{p(t)} d t \rightarrow \infty .
\end{aligned}
$$

Proposition 2 ([7]) The spaces $L^{p(t)}$ and $W_{T}^{1, p(t)}$ are separable and reflexive Banach spaces when $p^{-}>1$.

Proposition 3 ([7]) There is a continuous embedding $W_{T}^{1, p(t)} \hookrightarrow C\left([0, T] ; R^{N}\right)$; when $p^{-}>1$, it is a compact embedding.

Proposition 4 ([7]) For every $u \in \widetilde{W}_{T}^{1, p(t)}$, there is a constant $C$ independent of $u$ such that

$$
\|u\|_{\infty} \leq C\left|u^{\prime}\right|_{p(t)}
$$

Proposition 5 ([7]) Let $u=\bar{u}+\tilde{u} \in W_{T}^{1, p(t)}$, then the norm $\left|\tilde{u}^{\prime}\right|_{p(t)}$ is an equivalent norm on $\widetilde{W}_{T}^{1, p(t)}$ and $|\bar{u}|+\left|u^{\prime}\right|_{p(t)}$ is an equivalent norm on $W_{T}^{1, p(t)}$.

To prove the main theorem of the paper, we need the following generalized mountain pass theorem.

Lemma 1 ([20]) Let $E$ be a real Banach space with $E=V \oplus X$, where $V \neq 0$ is finite dimensional. Suppose $\varphi \in C^{1}(E, R)$ satisfies the $(P S)$ condition, and

(a) There exist $\rho, \alpha>0$ such that $\left.\varphi\right|_{\partial B_{\rho} \cap X} \geq \alpha$, where $B_{\rho}=\left\{u \in E \mid\|u\|_{E} \leq \rho\right\}, \partial B_{\rho}$ denotes the boundary of $B_{\rho}$;

(b) There exist $e \in \partial B_{1} \cap X$ and $r>\rho$ such that if $Q \equiv\left(\bar{B}_{r} \cap V\right) \oplus\{s e \mid 0 \leq s \leq r\}$, then $\left.\varphi\right|_{\partial Q} \leq \frac{\alpha}{2}$.

Then $\varphi$ possesses a critical value $c \geq \alpha$ which can be characterized as

$$
c \equiv \inf _{h \in \Gamma} \max _{u \in Q} \varphi(h(u))
$$

where $\Gamma=\{h \in C(\bar{Q}, E) \mid h=i d$ on $\partial Q\}$, and id denotes the identity operator.

\section{Proof of Theorem 1}

Define a functional $\varphi$ on $W_{T}^{1, p(t)}$ by

$$
\varphi(u)=\int_{0}^{T} \frac{1}{p(t)}\left|u^{\prime}(t)\right|^{p(t)} d t-\int_{0}^{T} F(t, u(t)) d t
$$

for each $u \in W_{T}^{1, p(t)}$. It follows from assumption $\left(F_{0}\right)$ that the functional $\varphi$ is continuously differentiable on $W_{T}^{1, p(t)}$. Moreover, we have

$$
\left\langle\varphi^{\prime}(u), v\right\rangle=\int_{0}^{T}\left(\left|u^{\prime}(t)\right|^{p(t)-2} u^{\prime}(t), v^{\prime}(t)\right) d t-\int_{0}^{T}(\nabla F(t, u(t)), v(t)) d t
$$

for all $u, v \in W_{T}^{1, p(t)}$. And it is well known (see [7]) that the problem of finding a $T$-periodic solution of system (1) is equal to that of finding the critical of functional $\varphi$. 
We shall apply Lemma 1 to $\varphi$ to prove Theorem 1 .

For the convenience to verify the $(P S)$ condition, we need the following lemma. The proof can be found in [7] or [8].

Lemma 2 Let $J(u)=\int_{0}^{T} \frac{1}{p(t)}\left|u^{\prime}(t)\right|^{p(t)} d t$ for $u \in W_{T}^{1, p(t)}$. Then $\left\langle J^{\prime}(u), v\right\rangle=\int_{0}^{T} \mid\left(\left|u^{\prime}(t)\right|^{p(t)-2} \times\right.$ $\left.u^{\prime}(t), v^{\prime}(t)\right) d t$ for all $u, v \in W_{T}^{1, p(t)}$. And $J^{\prime}$ is a mapping of type $\left(S_{+}\right)$, i.e., if $u_{n} \rightarrow u$ and $\limsup _{n \rightarrow \infty}\left\langle J^{\prime}\left(u_{n}\right)-J^{\prime}(u), u_{n}-u\right\rangle \leq 0$, then $\left\{u_{n}\right\}$ has a convergent subsequence in $W_{T}^{1, p(t)}$.

In the following lemma we will show that $\varphi$ satisfies the $(P S)$ condition.

Lemma 3 The functional $\varphi$ satisfies the (PS) condition, i.e., for every sequence $\left\{u_{n}\right\} \in$ $W_{T}^{1, p(t)},\left\{u_{n}\right\}$ has a convergent subsequence if

$$
\left\{\varphi\left(u_{n}\right)\right\} \quad \text { is bounded and } \varphi^{\prime}\left(u_{n}\right) \rightarrow 0 \quad \text { as } n \rightarrow \infty .
$$

Proof First we prove that $\left\{u_{n}\right\}$ is a bounded sequence in $W_{T}^{1, p(t)}$. Otherwise, $\left\{u_{n}\right\}$ would be unbounded. Passing to a subsequence, we may assume that $\left\|u_{n}\right\| \rightarrow \infty$. Let $w_{n}=\frac{u_{n}}{\left\|u_{n}\right\|}$, so that $\left\|w_{n}\right\|=1$. By Proposition 3, also passing to a subsequence, we can suppose that

$$
\begin{array}{ll}
w_{n} \rightarrow w & \text { weakly in } W_{T}^{1, p(t)}, \\
w_{n} \rightarrow w & \text { strongly in } C\left([0, T] ; R^{N}\right)
\end{array}
$$

as $n \rightarrow \infty$. Moreover, we have

$$
\bar{w}_{n}=\frac{1}{T} \int_{0}^{T} w_{n}(t) d t \rightarrow \frac{1}{T} \int_{0}^{T} w(t) d t=\bar{w}
$$

as $n \rightarrow \infty$. By (4) there exists a constant $C_{1}>0$ such that

$$
\begin{aligned}
& \int_{0}^{T}\left(\frac{\mu}{p(t)}-1\right)\left|u_{n}^{\prime}(t)\right|^{p(t)} d t \\
& \quad=\mu \varphi\left(u_{n}\right)-\left\langle\varphi^{\prime}\left(u_{n}\right), u_{n}\right\rangle+\int_{0}^{T}\left(\mu F\left(t, u_{n}(t)\right)-\left(\nabla F\left(t, u_{n}(t)\right), u_{n}(t)\right)\right) d t \\
& \quad \leq C_{1}\left(1+\left\|u_{n}\right\|\right)+\int_{0}^{T}\left(\mu F\left(t, u_{n}(t)\right)-\left(\nabla F\left(t, u_{n}(t)\right), u_{n}(t)\right)\right) d t
\end{aligned}
$$

Notice that $\left\|u_{n}\right\| \rightarrow \infty$, we have

$$
\begin{aligned}
\left(\frac{\mu}{p^{+}}-1\right) \int_{0}^{T}\left|w_{n}^{\prime}(t)\right|^{p(t)} d t & =\left(\frac{\mu}{p^{+}}-1\right) \int_{0}^{T} \frac{\left|u_{n}^{\prime}(t)\right|^{p(t)}}{\left\|u_{n}\right\|^{p(t)}} d t \\
& \leq \int_{0}^{T}\left(\frac{\mu}{p(t)}-1\right) \frac{\left|u_{n}^{\prime}(t)\right|^{p(t)}}{\left\|u_{n}\right\|^{p^{-}}} d t .
\end{aligned}
$$

So, we obtain

$$
\begin{aligned}
& \left(\frac{\mu}{p^{+}}-1\right) \int_{0}^{T}\left|w_{n}^{\prime}(t)\right|^{p(t)} d t \\
& \quad \leq \frac{C_{1}\left(1+\left\|u_{n}\right\|\right)}{\left\|u_{n}\right\|^{p^{-}}}+\int_{0}^{T} \frac{\left(\mu F\left(t, u_{n}(t)\right)-\left(\nabla F\left(t, u_{n}(t)\right), u_{n}(t)\right)\right)}{\left\|u_{n}\right\|^{p^{-}}} d t .
\end{aligned}
$$


In view of $\left(F_{0}\right)$ and $\left(F_{3}\right)$, there exists $\Omega_{0} \subset[0, T]$ with meas $\left(\Omega_{0}\right)=0$ such that

$$
|F(t, u)| \leq a(|u|) b(t), \quad \nabla|F(t, u)| \leq a(|u|) b(t)
$$

for all $u \in R^{N}$ and $t \in[0, T] \backslash \Omega_{0}$ and

$$
\limsup _{|u| \rightarrow \infty} \frac{\mu F(t, u)-(\nabla F(t, u), u)}{|u|^{p^{-}}} \leq 0
$$

uniformly for $t \in[0, T] \backslash \Omega_{0}$. This yields

$$
\limsup _{n \rightarrow \infty} \frac{\mu F\left(t, u_{n}(t)\right)-\left(\nabla F\left(t, u_{n}(t)\right), u_{n}(t)\right)}{\left\|u_{n}\right\|^{p^{-}}} \leq 0
$$

for $t \in[0, T] \backslash \Omega_{0}$. Otherwise, there exist $t_{0} \in[0, T] \backslash \Omega_{0}$ and a subsequence of $u_{n}$, still denoted by $u_{n}$, such that

$$
\limsup _{n \rightarrow \infty} \frac{\mu F\left(t_{0}, u_{n}\left(t_{0}\right)\right)-\left(\nabla F\left(t_{0}, u_{n}\left(t_{0}\right)\right), u_{n}\left(t_{0}\right)\right)}{\left\|u_{n}\right\|^{p^{-}}}>0
$$

If $\left\{u_{n}\left(t_{0}\right)\right\}$ is bounded, then there exists a positive constant $C_{2}$ such that $\left|u_{n}\left(t_{0}\right)\right| \leq C_{2}$ for all $n \in \mathbf{N}$. By (7) we find

$$
\begin{array}{r}
\frac{\mu F\left(t_{0}, u_{n}\left(t_{0}\right)\right)-\left(\nabla F\left(t_{0}, u_{n}\left(t_{0}\right)\right), u_{n}\left(t_{0}\right)\right)}{\left\|u_{n}\right\|^{p^{-}}} \\
\leq \frac{\left(\mu+C_{2}\right) \max _{0 \leq s \leq C_{2}} a(s) b\left(t_{0}\right)}{\left\|u_{n}\right\|^{-}} \rightarrow 0
\end{array}
$$

as $n \rightarrow \infty$, which contradicts (9). So, there is a subsequence of $u_{n}\left(t_{0}\right)$, still denoted by $u_{n}\left(t_{0}\right)$, such that $\left|u_{n}\left(t_{0}\right)\right| \rightarrow \infty$ as $n \rightarrow \infty$.

$$
\begin{aligned}
& \limsup _{n \rightarrow \infty} \frac{\mu F\left(t_{0}, u_{n}\left(t_{0}\right)\right)-\left(\nabla F\left(t_{0}, u_{n}\left(t_{0}\right)\right), u_{n}\left(t_{0}\right)\right)}{\left\|u_{n}\right\| p^{-}} \\
& \quad=\limsup _{n \rightarrow \infty} \frac{\mu F\left(t_{0}, u_{n}\left(t_{0}\right)\right)-\left(\nabla F\left(t_{0}, u_{n}\left(t_{0}\right)\right), u_{n}\left(t_{0}\right)\right)}{\left|u_{n}\left(t_{0}\right)\right|^{p^{-}}}\left|w_{n}\left(t_{0}\right)\right|^{p^{-}} \\
& \quad=\limsup _{n \rightarrow \infty} \frac{\mu F\left(t_{0}, u_{n}\left(t_{0}\right)\right)-\left(\nabla F\left(t_{0}, u_{n}\left(t_{0}\right)\right), u_{n}\left(t_{0}\right)\right)}{\left|u_{n}\left(t_{0}\right)\right|^{p^{-}}} \lim _{n \rightarrow \infty}\left|w_{n}\left(t_{0}\right)\right|^{p^{-}} \\
& \quad \leq 0 .
\end{aligned}
$$

This contradicts (9). Thus, (8) holds. From (6) and (8) we obtain

$$
\limsup _{n \rightarrow \infty}\left(\frac{\mu}{p^{+}}-1\right) \int_{0}^{T}\left|w_{n}^{\prime}(t)\right|^{p(t)} d t \leq 0 .
$$

Since $\mu>p^{+}$, we get

$$
\lim _{n \rightarrow \infty}\left(\frac{\mu}{p^{+}}-1\right) \int_{0}^{T}\left|w_{n}^{\prime}(t)\right|^{p(t)} d t=0
$$


Combining with (5), this yields

$$
w_{n} \rightarrow \bar{w} \quad \text { as } n \rightarrow \infty
$$

which means that

$$
w=\bar{w} \quad \text { and } \quad T|\bar{w}|=\|w\|=1 .
$$

Then we have

$$
u_{n}(t) \rightarrow \infty \quad \text { as } n \rightarrow \infty
$$

uniformly for a.e. $t \in[0, T]$. And we get from $\left(F_{1}\right),\left(F_{4}\right)$ and Fatou's lemma that

$$
\begin{aligned}
& \liminf _{n \rightarrow \infty} \int_{0}^{T} \frac{F\left(t, u_{n}(t)\right)}{\left\|u_{n}\right\| p^{+}} d t \\
& \quad \geq \int_{0}^{T} \liminf _{n \rightarrow \infty} \frac{F\left(t, u_{n}(t)\right)}{\left\|u_{n}\right\| p^{+}} d t \\
& \quad=\int_{0}^{T} \liminf _{n \rightarrow \infty} \frac{F\left(t, u_{n}(t)\right)}{\left|u_{n}(t)\right|^{p^{+}}}\left|w_{n}(t)\right|^{p^{+}} d t \\
& \geq \int_{\Omega} \liminf _{n \rightarrow \infty} \frac{F\left(t, u_{n}(t)\right)}{\left|u_{n}(t)\right|^{p^{+}}}\left|w_{0}\right|^{p^{+}} d t>0 .
\end{aligned}
$$

On the other hand, we have

$$
\begin{aligned}
\int_{0}^{T} \frac{F\left(t, u_{n}(t)\right)}{\left\|u_{n}\right\|^{p^{+}}} d t & =\int_{0}^{T} \frac{1}{p(t)} \frac{\left|u_{n}^{\prime}(t)\right|^{p(t)}}{\left\|u_{n}\right\|^{p^{+}}} d t-\frac{\varphi\left(u_{n}\right)}{\left\|u_{n}\right\|^{p^{+}}} \\
& \leq \frac{1}{p^{-}} \int_{0}^{T}\left|\frac{u_{n}^{\prime}(t)}{\left\|u_{n}\right\|}\right|^{p(t)} d t-\frac{\varphi\left(u_{n}\right)}{\left\|u_{n}\right\|^{p^{+}}} \\
& =\frac{1}{p^{-}} \int_{0}^{T}\left|w_{n}^{\prime}(t)\right|^{p(t)} d t-\frac{\varphi\left(u_{n}\right)}{\left\|u_{n}\right\|^{p^{+}}}
\end{aligned}
$$

Therefore, combining (4) and (10), we obtain that

$$
\liminf _{n \rightarrow \infty} \int_{0}^{T} \frac{F\left(t, u_{n}(t)\right)}{\left\|u_{n}\right\|^{p^{+}}} d t \leq 0
$$

which contradicts (11). Hence, $\left\{u_{n}\right\}$ is a bounded sequence in $W_{T}^{1, p(t)}$.

By Proposition 2 and Proposition 3, $\left\{u_{n}\right\}$ has a subsequence, again denoted by $\left\{u_{n}\right\}$, such that

$$
\begin{array}{ll}
u_{n} \rightarrow u & \text { weakly in } W_{T}^{1, p(t)}, \\
u_{n} \rightarrow u & \text { strongly in } C\left([0, T] ; R^{N}\right) .
\end{array}
$$

Now, we will show that $\left\{u_{n}\right\}$ has a subsequence convergent strongly to $u$ in $W_{T}^{1, p(t)}$. From Lemma 2 it suffices to prove that $\lim _{\sup _{n \rightarrow \infty}}\left\langle J^{\prime}\left(u_{n}\right)-J^{\prime}(u), u_{n}-u\right\rangle \leq 0$. 
It follows from Proposition 3 that $\max _{0 \leq t \leq T}\left|u_{n}(t)\right| \leq C_{3}\left\|u_{n}\right\|$, which implies

$$
\left|u_{n}(t)\right| \leq C_{4} \quad \text { for all } t \in[0, T]
$$

From (12), (13) and $\left(F_{0}\right)$, we get

$$
\begin{aligned}
& \left|\int_{0}^{T}\left(\nabla F\left(t, u_{n}(t)\right), u_{n}(t)-u(t)\right) d t\right| \\
& \quad \leq \int_{0}^{T}\left|\nabla F\left(t, u_{n}(t)\right)\right|\left|u_{n}(t)-u(t)\right| d t \\
& \quad \leq\left\|u_{n}-u\right\|_{\infty} \int_{0}^{T} a\left(\left|u_{n}(t)\right|\right) b(t) d t \\
& \quad \leq C_{5}\left\|u_{n}-u\right\|_{\infty} \int_{0}^{T} b(t) d t .
\end{aligned}
$$

Thus, from (12), we obtain

$$
\left|\int_{0}^{T}\left(\nabla F\left(t, u_{n}(t)\right), u_{n}(t)-u(t)\right) d t\right| \rightarrow 0 .
$$

By (4) and (13), we have

$$
\left\langle\varphi^{\prime}\left(u_{n}\right), u_{n}-u\right\rangle \rightarrow 0
$$

Then it follows from (14) and (15) that

$$
\begin{aligned}
\left\langle J^{\prime}\left(u_{n}\right), u_{n}-u\right\rangle & =\int_{0}^{T}\left(\left|u_{n}^{\prime}(t)\right|^{p(t)-2} u_{n}^{\prime}(t), u_{n}^{\prime}(t)-u^{\prime}(t)\right) d t \\
& =\left\langle\varphi^{\prime}\left(u_{n}\right), u_{n}-u\right\rangle+\int_{0}^{T}\left(\nabla F\left(t, u_{n}(t)\right), u_{n}(t)-u(t)\right) d t \\
& \rightarrow 0 \quad \text { as } n \rightarrow \infty .
\end{aligned}
$$

Moreover, since $J^{\prime}(u) \in\left(W_{T}^{1, p(t)}\right)^{*}$, we get $\left\langle\varphi^{\prime}(u), u_{n}-u\right\rangle \rightarrow 0$, which combined with (16) implies that

$$
\lim _{n \rightarrow \infty}\left\langle J^{\prime}\left(u_{n}\right)-J^{\prime}(u), u_{n}-u\right\rangle \leq 0
$$

Hence, from Lemma 2, $\left\{u_{n}\right\}$ has a subsequence convergent strongly to $u$ in $W_{T}^{1, p(t)}$. The proof of the lemma is completed.

The following result establishes the generalized mountain pass geometry for the functional $\varphi(u)$.

Lemma 4 Let $W_{T}^{1, p(t)}=R^{N} \oplus \widetilde{W}_{T}^{1, p(t)}, \mathbf{B}_{r}=\left\{u \in W_{T}^{1, p(t)} \mid\|u\| \leq r\right\}, \mathbf{S}_{r}=\widetilde{W}_{T}^{1, p(t)} \cap \partial \mathbf{B}_{r}$. Then there exist $\rho>0$ and $\alpha>0$ such that

$$
\inf _{u \in \mathbf{S}_{\rho}} \varphi(u)>\alpha .
$$


And there exist $r_{1}>0, r_{2}>\rho$ and $e \in \widetilde{W}_{T}^{1, p(t)}$ such that

$$
\sup _{u \in \partial \mathbf{Q}} \varphi(u) \leq 0
$$

where $\mathbf{Q}=\left\{u+s e \mid u \in R^{N} \cap \mathbf{B}_{r_{1}}, s \in\left[0, r_{2}\right]\right\}$.

Proof Firstly, we show that there exists $\rho>0$ such that $\inf _{u \in \mathbf{S}_{\rho}} \varphi(u)>0$. Let $C$ be the constant in Proposition 4. By condition $\left(F_{2}\right)$, we know that for any positive constant $\epsilon<\min \left\{C, \frac{1}{p^{+} T C^{p^{+}}}\right\}$, there exists $\delta \in(0, \epsilon)$ such that

$$
|F(t, u)| \leq \epsilon|u|^{p^{+}}
$$

for all $|u| \leq \delta$ and a.e. $t \in[0, T]$. Let $0<\rho \leq \frac{\delta}{C}$ and by Proposition 5 set $\mathbf{S}_{\rho}=\{u \in$ $\left.\left.\widetilde{W}_{T}^{1, p(t)}|| u^{\prime}\right|_{p(t)}=\rho\right\}$. By Proposition 4, we get $|u(t)| \leq C\left|u^{\prime}\right|_{p(t)}=C \rho=\delta$. Since $\rho<1$, then it follows from Proposition 1 and (17) that

$$
\begin{aligned}
\varphi(u) & =\int_{0}^{T} \frac{1}{p(t)}\left|u^{\prime}(t)\right|^{p(t)} d t-\int_{0}^{T} F(t, u(t)) d t \\
& \geq \frac{1}{p^{+}} \int_{0}^{T}\left|u^{\prime}(t)\right|^{p(t)} d t-\epsilon \int_{0}^{T}|u(t)|^{p^{+}} d t \\
& \geq \frac{1}{p^{+}}\left|u^{\prime}\right|_{p(t)}^{p^{+}}-\epsilon T C^{p^{+}}\left|u^{\prime}\right|_{p(t)}^{p^{+}} \\
& =\left(\frac{1}{p^{+}}-\epsilon T C^{p^{+}}\right) \rho^{p^{+}}=\alpha>0 .
\end{aligned}
$$

Secondly, we prove that there exist $r_{1}>0, r_{2}>\rho$ and $e \in \widetilde{W}_{T}^{1, p(t)}$ such that $\sup _{u \in \partial \mathbf{Q}} \varphi(u) \leq$ 0 . By $\left(F_{3}\right)$ and $\left(F_{4}\right)$ there exist constants $C_{6}>\max \{1, G\}, \eta>0$ and a subset of $\Omega$, still denoted by $\Omega$, with $|\Omega|=$ meas $(\Omega)>0$ such that

$$
\mu F(t, u)-(\nabla F(t, u), u) \leq \eta|u|^{p^{-}}
$$

and

$$
F(t, u)>\frac{2 \eta}{\mu-p^{-}}|u|^{p^{+}}
$$

for all $|u| \geq C_{6}$ and $t \in \Omega$. For $u \in R^{N} \backslash\{0\}$ and $t \in \Omega$, let $f(s)=F(t, s u)$ for all $s \geq \frac{C_{6}}{|u|}$. We deduce from (18) that

$$
\begin{aligned}
f^{\prime}(s) & =\frac{1}{s}(\nabla F(t, s u), s u) \\
& \geq \frac{\mu}{s} F(t, s u)-\eta s^{p^{-}-1}|u|^{p^{-}} \\
& =\frac{\mu}{s} f(s)-\eta s^{p^{-}-1}|u|^{p^{-}},
\end{aligned}
$$

which yields

$$
g(s)=f^{\prime}(s)-\frac{\mu}{s} f(s)+\eta s^{p^{-}-1}|u|^{p^{-}} \geq 0
$$


for all $s \geq \frac{C_{6}}{|u|}$. From (20) we have

$$
f(s)=\left(\int_{\frac{C_{6}}{|u|}}^{s} \frac{g(r)-\eta r^{p^{-}-1}|u|^{p^{-}}}{r^{\mu}} d r+\left(\frac{|u|}{C_{6}}\right)^{\mu} f\left(\frac{C_{6}}{|u|}\right)\right) s^{\mu}
$$

for all $s \geq \frac{C_{6}}{|u|}$. It follows from (21) and (20) that

$$
\begin{aligned}
f(s) & \geq\left(\left(\frac{|u|}{C_{6}}\right)^{\mu} f\left(\frac{C_{6}}{|u|}\right)+\frac{\eta|u|^{p^{-}}}{\left(\mu-p^{-}\right) s^{\mu-p^{-}}}-\frac{\eta|u|^{\mu}}{\left(\mu-p^{-}\right) C_{6}^{\mu-p^{-}}}\right) s^{\mu} \\
& \geq\left(F\left(t, \frac{C_{6}}{|u|} u\right)-\frac{\eta C_{6}^{p^{-}}}{\mu-p^{-}}\right)\left(\frac{|u|}{C_{6}}\right)^{\mu} s^{\mu} .
\end{aligned}
$$

Combining this with (19) yields

$$
\begin{aligned}
F(t, u) & =f(1) \geq\left(F\left(t, \frac{C_{6}}{|u|} u\right)-\frac{\eta C_{6}^{p^{-}}}{\mu-p^{-}}\right)\left(\frac{|u|}{C_{6}}\right)^{\mu} \\
& \geq\left(\frac{2 \eta C_{6}^{p^{+}-\mu}}{\mu-p^{-}}-\frac{\eta C_{6}^{p^{-}-\mu}}{\mu-p^{-}}\right)|u|^{\mu} \\
& \geq C_{7}|u|^{\mu}
\end{aligned}
$$

for all $|u| \geq C_{6}$ and $t \in \Omega$, where $C_{7}=\frac{\eta}{\mu-p^{-}}\left(\frac{2}{C_{6}^{\mu-p^{+}}}-\frac{1}{C_{6}^{\mu-p^{-}}}\right)>0$. So, notice that $F(t, u) \geq 0$, we get

$$
F(t, u) \geq C_{7}\left(|u|^{\mu}-C_{6}^{\mu}\right)=C_{7}|u|^{\mu}-C_{8}
$$

for all $u \in R^{N}$ and $t \in \Omega$.

Choose $e(t) \in \widetilde{W}_{T}^{1, p(t)}$ with $\|e(t)\|=1$ such that $e(t)=0$ for all $t \in[0, t] \backslash \Omega$. Therefore, one has

$$
\int_{\Omega} e(t) d t=\int_{0}^{T} e(t) d t-\int_{[0, t] \backslash \Omega} e(t) d t=0
$$

which implies that

$$
\int_{\Omega}(u, e(t)) d t=\int_{0}^{T}(u, e(t)) d t-\int_{[0, t] \backslash \Omega}(u, e(t)) d t=0
$$

for all $u \in R^{N}$. Let $\bar{W}_{T}^{1, p(t)}=R^{N} \oplus \operatorname{span}\{e(t)\}$. Since $\operatorname{dim}\left(\bar{W}_{T}^{1, p(t)}\right)<\infty$, all the norms are equivalent. For any $v=u+s e(t) \in \bar{W}_{T}^{1, p(t)}$, there exists a positive constant $K$ such that 
Denoting $E_{1}=\int_{0}^{T}\left|e^{\prime}(t)\right|^{p(t)} d t, E_{2}=\int_{\Omega}|e(t)|^{2} d t$, by (22), (23), (24) and (F $F_{1}$, we get

$$
\begin{aligned}
\varphi(u+s e) & =\int_{0}^{T} \frac{1}{p(t)}\left|s e^{\prime}(t)\right|^{p(t)} d t-\int_{0}^{T} F(t, u+s e(t)) d t \\
& \leq \frac{1}{p^{-}} \int_{0}^{T}\left|s e^{\prime}(t)\right|^{p(t)} d t-\int_{\Omega} F(t, u+s e(t)) d t \\
& \leq \frac{1}{p^{-}} \int_{0}^{T}\left|s e^{\prime}(t)\right|^{p(t)} d t-C_{7} \int_{\Omega}|u+s e(t)|^{\mu} d t+C_{8}|\Omega| \\
& \leq \frac{1}{p^{-}} \int_{0}^{T}\left|s e^{\prime}(t)\right|^{p(t)} d t-C_{7} K^{\mu}\left(\int_{\Omega}|u+s e(t)|^{2} d t\right)^{\frac{\mu}{2}}+C_{8}|\Omega| \\
& =\frac{1}{p^{-}} \int_{0}^{T}\left|s e^{\prime}(t)\right|^{p(t)} d t-C_{7} K^{\mu}\left(\int_{\Omega}\left(|u|^{2}+s^{2}|e(t)|^{2}\right) d t\right)^{\frac{\mu}{2}}+C_{8}|\Omega| \\
& \leq \frac{1}{p^{-}} \int_{0}^{T}\left|s e^{\prime}(t)\right|^{p(t)} d t-C_{7} K^{\mu}|u|^{\mu}|\Omega|^{\frac{\mu}{2}}-C_{7} K^{\mu} s^{\mu}\left|E_{2}\right|^{\frac{\mu}{2}}+C_{8}|\Omega| .
\end{aligned}
$$

Therefore, when $s>1$, we have

$$
\varphi(u+s e) \leq \frac{E_{1}}{p^{-}} s^{p^{+}}-C_{7} K^{\mu}\left|E_{2}\right|^{\frac{\mu}{2}} s^{\mu}+C_{8}|\Omega| .
$$

Since $\mu>p^{+}$, there exists $r_{2}>\max \{1, \rho\}$ such that

$$
\varphi(u+s e) \leq 0 \quad \text { for all } u \in R^{N} \text { and } s=r_{2}
$$

Moreover, for all $u \in R^{N}$ and $0 \leq s \leq r_{2}$, we have

$$
\varphi(u+s e) \leq \frac{E_{1} r_{2}^{p^{+}}}{p^{-}}-C_{7} K^{\mu}|u|^{\mu}|\Omega|^{\frac{\mu}{2}}+C_{8}|\Omega| .
$$

This deduces that

$$
\varphi(u+s e) \leq 0 \quad \text { when }|u|^{\mu} \geq \frac{E_{1} r_{2}^{p^{+}}+C_{8}|\Omega| p^{-}}{C_{7} K^{\mu}|\Omega|^{\frac{\mu}{2}} p^{-}}
$$

Let $u \in R^{N},|u| \geq 1$, from Proposition 4 , we know that

$$
|u|^{\mu} T=\int_{0}^{T}|u|^{\mu} d t \geq \int_{0}^{T}|u|^{p(t)} d t \geq|u|_{p(t)}^{p^{-}}
$$

So, let $r_{1}$ satisfy

$$
r_{1}^{p^{-}} \geq \max \left\{1, \frac{E_{1} r_{2}^{p^{+}}+C_{8}|\Omega| p^{-}}{C_{7} K^{\mu}|\Omega|^{\frac{\mu}{2}} p^{-}} T\right\}
$$

then, when $u \in R^{N},\|u\|=|u|_{p(t)}=r_{1}$, we obtain

$$
\varphi(u+s e) \leq 0 \quad \text { for all } s \in\left[0, r_{2}\right]
$$


On the other hand, if $s=0$, by $\left(F_{1}\right)$, we get

$$
\varphi(u+s e)=-\int_{0}^{T} F(t, u) d t \leq 0 \quad \text { for all } u \in R^{N} .
$$

Setting $\mathbf{Q}=\left\{u+s e \mid u \in R^{N} \cap \mathbf{B}_{r_{1}}, s \in\left[0, r_{2}\right]\right\}$, by (25),(26) and (27), we have

$$
\sup _{u \in \partial \mathbf{Q}} \varphi(u) \leq 0 .
$$

The proof of Lemma 4 is completed.

Proof of Theorem 1 By Lemma 3 and Lemma 4, applying Lemma 1, then $\varphi$ possesses a critical point $u(t)$ whose critical value $c$ satisfies $c \geq \alpha>0$. By $F_{1}$, we can see that $u(t)$ is nonconstant. Hence, problem (1) has at least one nonconstant $T$-periodic solution in $\mathbf{W}_{T}^{1, p(t)}$.

\section{Conclusions}

In this work, we have established an existence result for nonconstant periodic solutions of a class of second-order systems with $p(t)$-Laplacian. For $p(x)$ is a constant $p$, it is easy to see that the conditions and conclusion in Theorem 1 are the same as those in Theorem 1.4 in [6]. Thus Theorem 1 generalizes Theorem 1.4 in [6] and Theorem 1.1 in [5]. Furthermore, obviously, conditions $\left(F_{2}\right)$ and $\left(F_{3}\right)$ of Theorem 1 are weaker than $\left(A_{2}\right)$ and $\left(A_{3}\right)$ of Theorem 4.1 in [8]. Therefore, Theorem 1 extends Theorem 4.1 in [8].

Funding

This work is supported in part by the National Natural Foundation of China-NSAF (Grant No.11571092, No.11501165) and the Fundamental Research Funds for the Central Universities (2015B19414).

Availability of data and materials

Not applicable.

Ethics approval and consent to participate

Not applicable.

\section{Competing interests}

The authors declare that there is no conflict of interests regarding the publication of this article.

Consent for publication

Not applicable.

Authors' contributions

The authors have contributed equally and significantly in writing this article. All authors read and approved the final manuscript.

Author details

${ }^{1}$ College of Science, Nanjing University of Aeronautics and Astronautics, Nanjing, 210016, P.R. China. ${ }^{2}$ College of Science, China Pharmaceutical University, Nanjing, 211198, P.R. China. ${ }^{3}$ College of Science, Hohai University, Nanjing, 210098, P.R. China.

\section{Publisher's Note}

Springer Nature remains neutral with regard to jurisdictional claims in published maps and institutional affiliations. 


\section{References}

1. Zhikov, W: Averaging of functionals of the calculus of variations and elasticity theory. Math. USSR, Izv. 9, 33-66 (1987)

2. Rabinowitz, PH: Periodic solutions of Hamiltonian systems. Commun. Pure Appl. Math. 31, 157-184 (1978)

3. Schechter, M: Periodic non-autonomous second-order dynamical systems. J. Differ. Equ. 223, 290-302 (2006)

4. Schechter, M: Periodic solution of second order non-autonomous dynamical systems. Bound. Value Probl. 2006, $25104(2006)$

5. Wang, ZY, Zhang, JH, Zhang, ZT: Periodic solutions of second order non-autonomous Hamiltonian systems with local superquadratic potential. Nonlinear Anal. 70, 3672-3681 (2009)

6. Li, C, Agarwal, RP, Pu, Y, Tang, CL: Nonconstant periodic solutions for a class of ordinary p-Laplacian systems. Bound. Value Probl. 2016, 213 (2016)

7. Fan, XL, Fan, X: A Knobloch-type result for p(t)-Laplacian systems. J. Math. Anal. Appl. 282, 453-464 (2003)

8. Wang, XJ, Yuan, R: Existence of periodic solutions for p(t)-Laplacian systems. Nonlinear Anal. 70, 866-880 (2009)

9. Ayazoglu, R, Avci, M: Positive periodic solutions of nonlinear differential equations system with nonstandard growth. Appl. Math. Lett. 43, 5-9 (2015)

10. Zhang, SG: Periodic solutions for a class of second order Hamiltonian systems with $p(t)$-Laplacian. Bound. Value Probl. 2016, $211(2016)$

11. Zhang, L, Tang, $\mathrm{XH}$, Chen, J: Infinitely many periodic solutions for some differential systems with $p(t)$-Laplacian. Bound. Value Probl. 2011, 33 (2011)

12. Tan, Z, Fang, F: On superlinear $p(x)$-Laplacian problems without Ambrosetti and Rabinowitz condition. Nonlinear Anal. 75, 3902-3915 (2012)

13. Avci, CM: Existence and multiplicity of solutions for Dirichlet problems involving the $\mathrm{p}(\mathrm{x})$-Laplace operator. Electron. J. Differ. Equ. 14, 197 (2013)

14. Yucedag, Z: Existence of solutions for $\mathrm{p}(\mathrm{x})$-Laplacian equations without Ambrosetti-Rabinowitz type condition. Bull. Malays. Math. Sci. Soc. 38(3), 1023-1033 (2015)

15. Acerbi, E, Mingione, G: Regularity results for a class of functionals with nonstandard growth. Arch. Ration. Mech. Anal. $156,121-140(2001)$

16. Fan, $X L$, Zhao, D: On the spaces $L^{p(x)}$ and $W^{m, p(x)}$. J. Math. Anal. Appl. 263, 424-446 (2001)

17. Fan, $X L$, Shen, JS, Zhao, D: Sobolev embedding theorems for spaces $W^{k, p(x)}(\Omega)$. J. Math. Anal. Appl. 262, 749-760 (2001)

18. Fan, $\mathrm{XL}$, Zhao, $Y Z$, Zhao, D: Compact imbedding theorems with symmetry of Strauss-Lions type for the space $W^{1, p(x)}(\Omega)$. J. Math. Anal. Appl. 225, 333-348 (2001)

19. Edmunds, DE, Rakosnik, J: Sobolev embeddings with variable exponent. Math. Nachr. 246-247, 53-67 (2002)

20. Rabinowitz, PH: Minimax methods in critical point theory with applications to differential equations. In: CBMS Reg. Conf. Ser. in Math., vol. 65. Am. Math. Soc., Providence (1986)

\section{Submit your manuscript to a SpringerOpen ${ }^{\circ}$ journal and benefit from:}

- Convenient online submission

- Rigorous peer review

- Open access: articles freely available online

- High visibility within the field

- Retaining the copyright to your article

Submit your next manuscript at $>$ springeropen.com 